\title{
Determining the Role of Bacteriophages in the Virulence of Streptococcus agalactiae ${ }^{\dagger}$
}

\author{
Melody N. Neely *, Caitlin Sar Wiafe-Kwakye and Sally D. Molloy \\ Department of Molecular and Biomedical Sciences, University of Maine, 5835 Hitchner Hall, Orono, ME \\ 04469, USA; caitlin.tetteh@maine.edu (C.S.W.-K.); sally.dixon@maine.edu (S.D.M.) \\ * Correspondence: melody.neely@maine.edu \\ + Presented at Viruses 2020-Novel Concepts in Virology, Barcelona, Spain, 5-7 February 2020. \\ Published: 8 June 2020
}

\begin{abstract}
Bacteriophages (phage) are viruses that specifically infect bacteria and contribute to their evolution. Nearly all bacterial pathogens contain integrated phage, or prophage, within their genome, and many phage genes are associated with bacterial virulence and/or bacterial fitness. Prophages that are associated with the virulence of human pathogens include those found in Vibrio cholerae, Salmonella sp., Staphylococcus aureus, and Streptococcus pyogenes. Streptococcus agalactiae (Group B streptococcus or GBS) also contain prophages and, in many cases, multiple prophages in a single strain. GBS is an opportunistic pathogen that is found associated with the mucus membranes of the genitourinary tract in about $25 \%$ of the adult population. However, if GBS colonizes an immunocompromised individual (such as a neonate or pregnant mother), it can invade and survive in the bloodstream, resulting in serious morbidity and mortality. Unfortunately, treatment of neonates with antibiotics results in disruption of their normal gut microflora and has major effects on the development of their immune system. In order to address the threat of GBS to newborns, we need to fully understand the mechanisms by which this pathogen causes disease. We hypothesize that phage integration into the S. agalactiae genome may enhance pathogenicity and play a role in neonatal-associated infection. Through analysis of the genome sequences of seven $S$. agalactiae strains, we detected intact phage sequences in three out of the seven strains, which are A909, CNCTC 10/84, and 2603V/R. We have successfully cured the prophage, Callidus, from the CNCTC10/84 genome by overexpression of the excise gene. Comparative analyses between the WT lysogen and the cured strain show differences in chain length, cell morphology and virulence using a well-established zebrafish infectious disease model. Understanding GBS virulence mechanisms is key to developing novel, effective treatments and vaccines for GBS.
\end{abstract}

Keywords: bacteriophage; prophage; streptococcus; virulence; zebrafish

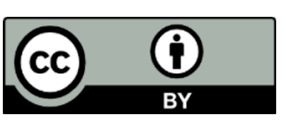

(C) 2020 by the authors. Licensee MDPI, Basel, Switzerland. This article is an open access article distributed under the terms and conditions of the Creative Commons Attribution (CC BY) license (http://creativecommons.org/licenses/by/4.0/). 